\title{
ON THE COMPLEXITY OF FINDING A NECESSARY AND SUFFICIENT CONDITION FOR BLASCHKE-OSCILLATORY EQUATIONS
}

\author{
JANNE HEITTOKANGAS AND ATTE REIJONEN \\ Department of Physics and Mathematics, University of Eastern Finland, \\ P.O. Box 111, 80101 Joensuu, Finland \\ e-mails: janne.heittokangas@uef.fi, atte.reijonen@uef.fi
}

(Received 27 September 2013; revised 12 February 2014; accepted 4 March 2014; first published online 17 December 2014)

\begin{abstract}
If $A(z)$ belongs to the Bergman space $B^{\frac{1}{2}}$, then the differential equation $f^{\prime \prime}+A(z) f=0$ is Blaschke-oscillatory, meaning that the zero sequence of every nontrivial solution satisfies the Blaschke condition. Conversely, if $A(z)$ is analytic in the unit disc such that the differential equation is Blaschke-oscillatory, then $A(z)$ almost belongs to $B^{\frac{1}{2}}$. It is demonstrated that certain "nice" Blaschke sequences can be zero sequences of solutions in both cases when $A \in B^{\frac{1}{2}}$ or $A \notin B^{\frac{1}{2}}$. In addition, no condition regarding only the number of zeros of solutions is sufficient to guarantee that $A \in B^{\frac{1}{2}}$.
\end{abstract}

2010 Mathematics Subject Classification. Primary 34M10; Secondary 30J10.

1. Introduction. In 1982, Pommerenke [17] proved that if $A(z)$ is analytic in the unit disc $\mathbb{D}$ (denoted by $A \in H(\mathbb{D})$ for short) and

$$
\int_{\mathbb{D}}|A(z)|^{\frac{1}{2}} d m(z)<\infty
$$

where $d m(z)=r d r d \theta$ is the Lebesgue area measure, then all solutions of

$$
f^{\prime \prime}+A(z) f=0
$$

belong to the Nevanlinna class $N$ [4]. This in turn implies that (2) is Blaschke-oscillatory, meaning that the zero sequence $\left\{z_{n}\right\}$ of any nontrivial solution of (2) satisfies the Blaschke condition

$$
\sum_{n}\left(1-\left|z_{n}\right|\right)<\infty
$$

The converse direction is considered in [10, Theorems 2 and 4 (b)]: If $A \in H(\mathbb{D})$ and if either (2) is Blaschke-oscillatory or (2) possesses a nontrivial solution in $N$, then

$$
\int_{D(0, r)}|A(z)|^{\frac{1}{2}} d m(z)=O\left(\log ^{2} \frac{e}{1-r}\right), \quad r \rightarrow 1^{-} .
$$


Moreover, if the solution $f \in N$ has the property that $f^{\prime} \in N$, then (4) improves to

$$
\int_{D(0, r)}|A(z)|^{\frac{1}{2}} d m(z)=O\left(\log \frac{e}{1-r}\right), \quad r \rightarrow 1^{-} .
$$

The problem of finding a necessary and sufficient condition for (2) to be Blaschkeoscillatory is still open. To illustrate the complexity of this problem, and also to obtain partial results in this direction, we will concentrate on prescribed zero sequences.

In 1959, Šeda $[\mathbf{1 2}, \mathbf{1 9}]$ proved that if $\left\{z_{n}\right\}_{n=1}^{\infty}$ is a given sequence of pairwise distinct points in $\mathbb{C}$ with no finite limit points, then there is an entire function $A(z)$ such that the differential equation (2) has a solution with zeros precisely at the points $z_{n}$. Here, we consider prescribed Blaschke sequences having certain constraints. For example, we say that an arbitrary sequence $\left\{z_{n}\right\}_{n=1}^{\infty}$ in $\mathbb{D}$ is separated, if

$$
\delta:=\inf _{n \neq k}\left|\frac{z_{n}-z_{k}}{1-\bar{z}_{n} z_{k}}\right|>0,
$$

and uniformly separated, if

$$
\delta_{u}:=\inf _{k} \prod_{n \neq k}\left|\frac{z_{n}-z_{k}}{1-\bar{z}_{n} z_{k}}\right|>0 .
$$

It is known that a uniformly separated sequence is a Blaschke sequence, while a separated sequence need not be one.

The convergence condition (3) guarantees that the corresponding Blaschke product

$$
B(z)=\prod_{n=1}^{\infty} \frac{\left|z_{n}\right|}{z_{n}} \frac{z_{n}-z}{1-\bar{z}_{n} z}
$$

converges in compact subsets of $\mathbb{D}$, and hence is an analytic function with zeros precisely at the points $z_{n}$. Since, by [2, Theorem 3], a uniformly separated sequence is interpolating, a Blaschke product with a uniformly separated zero sequence is known as interpolating. At times (3) needs to be strengthened, for example, to

$$
\sum_{n}\left(1-\left|z_{n}\right|\right)^{\alpha}<\infty
$$

where $\alpha \in(0,1]$. For example, if (8) holds for $\alpha \in(0,1 / 2)$, then the corresponding Blaschke product $B$ has the property that $B^{\prime}$ belongs to the Hardy space $H^{1-\alpha}$, and hence to $N$ [18, Theorem 2].

For $p>0$, the Bergman space $B^{p}$ consists of all functions $f \in H(\mathbb{D})$ satisfying

$$
\int_{\mathbb{D}}|f(z)|^{p} d m(z)<\infty .
$$

The discussion above leads us to consider the following problem.

Problem. Which assumptions on a given Blaschke sequence $\left\{z_{n}\right\}_{n=1}^{\infty}$ in $\mathbb{D}$ will guarantee that there exists a function $A \in B^{\frac{1}{2}}$ such that the differential equation (2) has a solution with zeros precisely at the points $z_{n}$ ? 
Clearly the zeros must be pairwise distinct and the possible limit points must be on $\partial \mathbb{D}$. Assuming, for example, that $\left\{z_{n}\right\}_{n=1}^{\infty}$ is separated will guarantee both requirements. We have the following partial result.

THEOREM 1 ([10], Theorem 19). Let $\left\{z_{n}\right\}_{n=1}^{\infty}$ be a uniformly separated sequence of nonzero points in $\mathbb{D}$ satisfying the condition (8) for some $\alpha \in(0,1)$. Then there exists a function $A \in B^{\frac{1}{2}}$ such that the differential equation (2) has a solution with zeros precisely at the points $z_{n}$.

We sketch the proof as follows. Take $f=B e^{g}$ for a candidate solution, where $B$ is a Blaschke product with zeros at the points $z_{n}$, and the function $g \in H(\mathbb{D})$ has the interpolation property

$$
g^{\prime}\left(z_{n}\right)=-\frac{B^{\prime \prime}\left(z_{n}\right)}{2 B^{\prime}\left(z_{n}\right)}, \quad n \in \mathbb{N}
$$

The latter guarantees that

$$
A(z)=-\frac{B^{\prime \prime}(z)}{B(z)}-2 g^{\prime}(z) \frac{B^{\prime}(z)}{B(z)}-g^{\prime}(z)^{2}-g^{\prime \prime}(z)
$$

is analytic in $\mathbb{D}$, and that $f$ solves (2). After a suitable $g$ is found, then the growth of $A(z)$ in $(10)$ is estimated $[\mathbf{1 0}$, p. 78].

Theorem 1 in the case $\alpha=1$ extends to the following result.

THEOREM 2. Let $\left\{z_{n}\right\}_{n=1}^{\infty}$ be a uniformly separated sequence of nonzero points in $\mathbb{D}$. Then there exists a function $A \in H(\mathbb{D})$ satisfying (5) such that the differential equation (2) has a solution with zeros precisely at the points $z_{n}$.

Although not entirely removable, the assumption (8) for $\alpha \in(0,1)$ in Theorem 1 can be slightly weakened to

$$
\sum_{n} h\left(1-\left|z_{n}\right|\right)<\infty
$$

where $h(x)$ is a positive continuous function on $(0,1)$ tending to zero as $x \rightarrow 0^{+}$, and satisfies the following conditions:

(i) $h(x) / x$ is decreasing and $h(x)$ is increasing on $(0,1)$;

(ii) $\int_{0}^{1}(1-r)^{-\frac{1}{2}} h(1-r)^{-\frac{1}{2}} r d r<\infty$.

Then the choice $h(x)=x \log ^{p} \frac{e^{p}}{x}, p>2$, shows that the following result is an improvement of Theorem 1 .

THEOREM 3. Let $h(t)$ be a function as above, and let $\left\{z_{n}\right\}_{n=1}^{\infty}$ be a uniformly separated sequence of nonzero points in $\mathbb{D}$ satisfying (11). Then there exists a function $A \in B^{\frac{1}{2}}$ such that the differential equation (2) has a solution with zeros precisely at the points $z_{n}$.

Next we turn our attention to the separation of the zero sequences. If $\left\{z_{n}\right\}_{n=1}^{\infty}$ is a union of two exponential sequences [4, p. 156] approaching pairwise to one another exponentially, then it is proved in [8, Theorem 5] that $A(z)$ cannot belong to the Korenblum space $\mathcal{A}^{-\infty}[\mathbf{9}$, p. 110]. In particular, both of the two component sequences are uniformly separated, and yet all solutions of (2) are "far away" from the class $N$ as being of infinite order of growth. 
In the recent study [3], it is illustrated that the growth of $A(z)$ within the Korenblum space is restricted by the (weighted) separation of the zeros of any solution, and conversely. Here, we assume the usual separation, but require the separation constant $\delta \in(0,1)$ in $(6)$ to be close enough to the constant one in terms of

$$
(2 \pi+1) \frac{\sqrt{1-\delta}}{(1-\sqrt{1-\delta})^{2}}<1 .
$$

This makes $\left\{z_{n}\right\}_{n=1}^{\infty}$ to be interpolating for some Bergman space $B^{p}$ with $p>1[\mathbf{5}$, p. 192]. Now the assumption on uniform separation in Theorem 1 can be relaxed at the expense of strengthening the Blaschke condition.

THEOREM 4. Let $\left\{z_{n}\right\}_{n=1}^{\infty}$ be a separated sequence of nonzero points in $\mathbb{D}$ such that the separation constant $\delta$ in (6) satisfies (12). Suppose in addition that (8) holds for $\alpha \in(0,1 / 2]$. Then there exists a function $A \in B^{\frac{1}{2}}$ such that the differential equation (2) has a solution with zeros precisely at the points $z_{n}$.

REMARK. The assumption (12) in Theorem 4 can in fact be replaced by a weaker condition that the upper uniform density $D^{+}$of $\left\{z_{n}\right\}_{n=1}^{\infty}$ is less than one. See [5, pp. 171-172] for more details.

So far we have concentrated in one prescribed zero sequence only. Next we will widen our point of view to concern all solutions. First, an example of a nonoscillatory differential equation of the form (2) is constructed in [10, Section 4.3] such that all nontrivial solutions of (2) are of unbounded characteristic. In this case it is clear that $A \notin B^{\frac{1}{2}}[17]$. Second, by a more careful analysis on an example, which can be found in $[10,13]$, we will prove that the solutions of (2) can have infinite uniformly separated and sparse zero sequences even if $A \notin B^{\frac{1}{2}}$. This result also shows that the requirement $\alpha \in(0,1 / 2]$ in Theorem 4 is essential.

THEOREM 5. There exists a function $A \in H(\mathbb{D})$ with $A \notin B^{\frac{1}{2}}$ such that the differential equation (2) has the following properties:

(a) There exists a zero-free solution base.

(b) There are solutions with infinitely many zeros.

(c) Every infinite zero sequence satisfies (8) for every $\alpha \in(1 / 2,1]$.

(d) Every infinite zero sequence is uniformly separated.

(e) There are infinite separated zero sequences whose separation constant $\delta$ satisfies (12).

(f) Every solution and all of their derivatives belong to $N$.

Note that even if the differential equation (2) is disconjugate, it is still possible that $A \notin B^{\frac{1}{2}}$. This can be seen by means of the gap series

$$
g(z)=\sum_{n=0}^{\infty}\left(\frac{2^{n}}{n}\right)^{2} z^{2^{n}}, \quad z \in \mathbb{D} .
$$

First, by [1, Proposition 2.1], we infer $g \notin B^{\frac{1}{2}}$. Second, using [20, Theorem 1 (II)] together with the standard representation

$$
g(z)=g(0)+\int_{0}^{z} g^{\prime}(\xi) d \xi
$$


yields $g \in H_{2}^{\infty}$. Finally, denoting $A(z)=g(z) /\|g\|_{H_{2}^{\infty}}$, we see that (2) is disconjugate by the proof of [16, Theorem I], while $A \notin B^{\frac{1}{2}}$.

Summarizing, we conclude that no condition regarding only the number of zeros of solutions of (2) is sufficient to guarantee that $A \in B^{\frac{1}{2}}$.

2. Proof of Theorem 2. One of the key results in proving Theorem 1 is

$$
\int_{\mathbb{D}}\left|\frac{B^{(k)}(z)}{B(z)}\right|^{\frac{1}{k}} d m(z)<\infty
$$

valid for an interpolating Blaschke product $B$ with zeros $\left\{z_{n}\right\}$ satisfying (8) for some $\alpha \in(0,1)[10$, Theorem 15]. We will require a variant of this result.

LEMMA 1. Let B be an interpolating Blaschke product. Then

$$
\int_{D(0, r)}\left|\frac{B^{(k)}(z)}{B(z)}\right|^{\frac{1}{k}} d m(z)=O\left(\log \frac{e}{1-r}\right), \quad r \rightarrow 1^{-},
$$

for all $k \in \mathbb{N}$.

Proof. If $B$ is an arbitrary Blaschke product, then Cauchy's formula yields

$$
\left|B^{(k)}(z)\right|=O\left((1-|z|)^{-k}\right), \quad|z| \rightarrow 1^{-},
$$

for every $k \in \mathbb{N}$. In particular,

$$
\int_{0}^{2 \pi}\left|B^{(k)}\left(r e^{i \theta}\right)\right|^{\frac{1}{k}} d \theta=O\left(\frac{1}{1-r}\right), \quad k \in \mathbb{N} .
$$

Suppose that $B$ is interpolating, and denote the pseudo-hyperbolic disc with centre $a \in \mathbb{D}$ and radius $r \in(0,1)$ by

$$
\Delta(a, r)=\left\{z \in \mathbb{D}:\left|\varphi_{a}(z)\right|<r\right\},
$$

where $\varphi_{a}(z)=(a-z) /(1-\bar{a} z)$. Let $K=\bigcup_{n \in \mathbb{N}} \Delta\left(z_{n}, \delta_{u} / 2\right)$, where $\left\{z_{n}\right\}_{n=1}^{\infty}$ is the zero sequence of $B$ and $\delta_{u}$ is the interpolation constant defined in (7). Now, by using (14) instead of [7, Theorem 1.1] in the proof of [10, Theorem 15], we obtain

$$
\int_{D(0, r) \backslash K}\left|\frac{B^{(k)}(z)}{B(z)}\right|^{\frac{1}{k}} d m(z)=O\left(\log \frac{e}{1-r}\right), \quad r \rightarrow 1^{-} .
$$

Integration over the discs $\Delta\left(z_{n}, \delta_{u} / 2\right)$ can be done in a similar way as in the proof of [10, Theorem 15]. Thus the assertion follows.

We proceed to prove Theorem 2. Let $B$ be an interpolating Blaschke product having zeros at the points $z_{n}$. By the proof of [11, Theorem 4.2], we can find $g \in H(\mathbb{D})$ satisfying (9). Indeed, the derivative of $g$ is given by

$$
g^{\prime}(z)=\sum_{k=1}^{\infty} \sigma_{k} \frac{C_{k}(z)}{B^{\prime}\left(z_{k}\right)} \frac{\left|z_{k}\right|^{2}-1}{\left(1-\bar{z}_{k} z\right)^{2}}
$$


where

$$
C_{k}(z)=B(z) \frac{1-\bar{z}_{k} z}{z_{k}-z} \quad \text { and } \quad \sigma_{k}=-\frac{B^{\prime \prime}\left(z_{k}\right)}{2 B^{\prime}\left(z_{k}\right)} .
$$

Now $f=B e^{g}$ solves (2), where $A(z)$ is given by (10) and belongs to $H(\mathbb{D})$.

Next, we proceed to prove that

$$
\int_{D(0, r)}\left|g^{\prime}(z)\right| d m(z)=O\left(\log \frac{e}{1-r}\right), \quad r \rightarrow 1^{-},
$$

and

$$
\int_{D(0, r)}\left|g^{\prime \prime}(z)\right|^{\frac{1}{2}} d m(z)=O\left(\log \frac{e}{1-r}\right), \quad r \rightarrow 1^{-} .
$$

Following [11], we know that there exist constants $M_{1}, M_{2}>0$ such that

$$
\left|\sigma_{k}\right| \leq \frac{M_{1}}{1-\left|z_{k}\right|^{2}} \quad \text { and } \quad \frac{1}{\left|B^{\prime}\left(z_{k}\right)\right|} \leq M_{2}\left(1-\left|z_{k}\right|^{2}\right)
$$

for all $k \in \mathbb{N}$. Hence

$$
\left|g^{\prime}(z)\right| \leq R \sum_{k=1}^{\infty} \frac{1-\left|z_{k}\right|^{2}}{\left|1-\bar{z}_{k} z\right|^{2}}
$$

where the constant $R>0$ is independent of $z$, and so

$$
\begin{aligned}
\int_{0}^{2 \pi}\left|g^{\prime}\left(r e^{i \theta}\right)\right| d \theta & \leq R \sum_{k=1}^{\infty} \frac{1-\left|z_{k}\right|^{2}}{1-\left|z_{k}\right|^{2} r^{2}} \int_{0}^{2 \pi} \frac{1-\left|z_{k}\right|^{2} r^{2}}{\left|1-\bar{z}_{k} t e^{i \theta}\right|^{2}} d \theta \\
& \leq 2 \pi R \sum_{k=1}^{\infty} \frac{1-\left|z_{k}\right|^{2}}{1-\left|z_{k}\right|^{2} r^{2}}=O\left(\frac{1}{1-r}\right), \quad r \rightarrow 1^{-}
\end{aligned}
$$

This implies (17). Now the Hölder inequality and the proof of [4, Theorem 5.5] yield

$$
\int_{0}^{2 \pi}\left|g^{\prime \prime}\left(r e^{i \theta}\right)\right|^{\frac{1}{2}} d \theta \leq(2 \pi)^{\frac{1}{2}}\left(\int_{0}^{2 \pi}\left|g^{\prime \prime}\left(r e^{i \theta}\right)\right| d \theta\right)^{\frac{1}{2}}=O\left(\frac{1}{1-r}\right), \quad r \rightarrow 1^{-},
$$

and so (18) follows.

Finally, by (10) it is clear that

$$
|A(z)|^{\frac{1}{2}} \leq\left|\frac{B^{\prime \prime}(z)}{B(z)}\right|^{\frac{1}{2}}+\sqrt{2}\left|g^{\prime}(z)\right|^{\frac{1}{2}}\left|\frac{B^{\prime}(z)}{B(z)}\right|^{\frac{1}{2}}+\left|g^{\prime}(z)\right|+\left|g^{\prime \prime}(z)\right|^{\frac{1}{2}} .
$$

Thus, the assertion follows by the Hölder inequality, (17), (18) and Lemma 1.

3. Proof of Theorem 3. We begin by introducing two lemmas in which $h(t)$ is a positive continuous function defined on $(0,1)$ and tends to zero as $t \rightarrow 0^{+}$. 
Lemma 2. Let $k \in \mathbb{N}$, and let $B$ be a Blaschke product formed with zeros $\left\{z_{n}\right\}_{n=1}^{\infty}$ satisfying (11) such that $h(x) / x$ is decreasing and $h(x)$ is increasing on $(0,1)$, and that

$$
\int_{0}^{1}(1-r)^{\frac{1-k}{k}} h(1-r)^{-\frac{1}{k}} r d r<\infty
$$

Then

$$
\int_{\mathbb{D}}\left|B^{(k)}\left(r e^{i \theta}\right)\right|^{\frac{1}{k}} d m(z)<\infty
$$

Proof. By [6, Theorem 3.1] the assertion holds for $k=1$. Hence, by $k-1$ applications of the proof of $[4$, Theorem 5.5], we can easily see that

$$
\int_{0}^{2 \pi}\left|B^{(k)}\left(r e^{i \theta}\right)\right| d \theta=O\left(\frac{1}{(1-\rho)^{k-1} h(1-\rho)}\right)
$$

for $\rho \in(r, 1)$. Thus the Hölder inequality yields

$$
\begin{aligned}
\int_{0}^{2 \pi}\left|B^{(k)}\left(r e^{i \theta}\right)\right|^{\frac{1}{k}} d \theta & \leq(2 \pi)^{\frac{1}{k}}\left(\int_{0}^{2 \pi}\left|B^{(k)}\left(r e^{i \theta}\right)\right| d \theta\right)^{\frac{1}{k}} \\
& =O\left(\frac{1}{(1-\rho)^{\frac{k-1}{k}} h(1-\rho)^{\frac{1}{k}}}\right)
\end{aligned}
$$

and so the assertion follows.

REMARK. As noted in [6], since our sequences already satisfy the Blaschke condition, the condition (11) provides with further information on the rate of increase of the zeros only if $h(x) \geq x$ as $x \rightarrow 0^{+}$. This means that if (22) is valid for some $k \in \mathbb{N}$, then (22) is valid when $k$ is replaced with any integer $j \in\{1, \ldots, k\}$.

By using Lemma 2, we can rewrite the proof of [10, Theorem 15], and achieve the following result.

Lemma 3. Let $B$ and $h$ be as in Lemma 2, and, in addition, suppose that $B$ is interpolating. Then (13) holds.

We proceed to prove Theorem 3. Let $B$ be an interpolating Blaschke product having zeros at the points $z_{n}$ satisfying (11). As in the proof of Theorem 2, we can find a function $g \in H(\mathbb{D})$ such that $f=B e^{g}$ solves (2), where $A(z)$ is given by (10) and belongs to $H(\mathbb{D})$.

By (20) and [6, Lemma 2.1], we have

$$
\int_{0}^{2 \pi}\left|g^{\prime}(z)\right| d \theta \leq R \sum_{k=1}^{\infty} \frac{1-\left|z_{k}\right|^{2}}{1-\left|z_{k}\right|^{2} r^{2}} \int_{0}^{2 \pi} \frac{1-\left|z_{k}\right|^{2} r^{2}}{\left|1-\bar{z}_{k} r e^{i \theta}\right|^{2}} d \theta=\frac{2 \pi R}{h(1-r)}
$$


Hence, the Hölder inequality and the proof of [4, Theorem 5.5] yield

$$
\begin{aligned}
\int_{0}^{2 \pi}\left|g^{\prime \prime}(z)\right|^{\frac{1}{2}} d \theta & \leq(2 \pi)^{\frac{1}{2}}\left(\int_{0}^{2 \pi}\left|g^{\prime \prime}(z)\right|^{\frac{1}{2}} d \theta\right)^{\frac{1}{2}} \\
& =O\left(\frac{1}{(1-r)^{\frac{1}{2}} h(1-r)^{\frac{1}{2}}}\right), \quad r \rightarrow 1^{-}
\end{aligned}
$$

Using the condition (ii) related to (11), we obtain $g^{\prime} \in B^{1}$ and $g^{\prime \prime} \in B^{\frac{1}{2}}$. Thus the assertion follows by (21), the Hölder inequality and Lemma 3.

4. Proof of Theorem 4. We require a new lemma on logarithmic derivatives of noninterpolating Blaschke products.

Lemma 4. Suppose that $B$ is a Blaschke product with a zero sequence $\left\{z_{n}\right\}_{n=1}^{\infty}$. Then (13) holds if

$$
\sum_{n=1}^{\infty}\left(1-\left|z_{n}\right|\right) \log \frac{e}{1-\left|z_{n}\right|}<\infty
$$

in the case $k=1$, and if (8) holds for $\alpha \in(0,1 / k]$ in the case $k \geq 2$.

Proof. Since

$$
\left|\frac{B^{\prime}(z)}{B(z)}\right| \leq \sum_{n=1}^{\infty} \frac{1-\left|z_{n}\right|^{2}}{\left|1-\bar{z}_{n} z\right|\left|z_{n}-z\right|}=\sum_{n=1}^{\infty}\left|\frac{\varphi_{z_{n}}^{\prime}(z)}{\varphi_{z_{n}}(z)}\right|,
$$

we have

$$
\begin{aligned}
\int_{\mathbb{D}}\left|\frac{B^{\prime}(z)}{B(z)}\right| d m(z) & \leq \sum_{n=1}^{\infty} \int_{\mathbb{D} \backslash \Delta\left(z_{n}, \frac{1}{2}\right)}\left|\frac{\varphi_{z_{n}}^{\prime}(z)}{\varphi_{z_{n}}(z)}\right| d m(z)+\sum_{n=1}^{\infty} \int_{\Delta\left(z_{n}, \frac{1}{2}\right)}\left|\frac{\varphi_{z_{n}}^{\prime}(z)}{\varphi_{z_{n}}(z)}\right| d m(z) \\
& =: S_{1}+S_{2},
\end{aligned}
$$

where $\Delta(a, r)$ is the pseudo-hyperbolic disc defined in (15). By the condition (24), it follows that

$$
\begin{aligned}
S_{1} & \leq 2 \sum_{n=1}^{\infty} \int_{\mathbb{D}}\left|\varphi_{z_{n}}^{\prime}(z)\right| d m(z) \\
& =2 \sum_{n=1}^{\infty} \int_{0}^{1} \frac{1-\left|z_{n}\right|^{2}}{1-\left|z_{n}\right|^{2} r^{2}}\left(\int_{0}^{2 \pi} \frac{1-\left|z_{n}\right|^{2} r^{2}}{\left|1-\bar{z}_{n} r e^{i \theta}\right|} d \theta\right) r d \theta d r \\
& =4 \pi \sum_{n=1}^{\infty}\left(1-\left|z_{n}\right|^{2}\right) \int_{0}^{1} \frac{r d r}{1-\left|z_{n}\right|^{2} r^{2}} \\
& \leq C_{1} \sum_{n=1}^{\infty}\left(1-\left|z_{n}\right|\right) \log \frac{e}{1-\left|z_{n}\right|}<\infty
\end{aligned}
$$


for some constant $C_{1}>0$. On the other hand, by a change of variable, we have

$$
\begin{aligned}
S_{2} & \leq \sum_{n=1}^{\infty} \int_{\Delta\left(z_{n}, \frac{1}{2}\right)} \frac{1-\left|z_{n}\right|^{2}}{\left(1-\left|z_{n}\right|\right)^{2}\left|\varphi_{z_{n}}(z)\right|} d m(z) \\
& \leq 2 \sum_{n=1}^{\infty} \frac{1}{1-\left|z_{n}\right|} \int_{\Delta\left(z_{n}, \frac{1}{2}\right)} \frac{d m(z)}{\left|\varphi_{z_{n}}(z)\right|} \\
& =2 \sum_{n=1}^{\infty} \frac{1}{1-\left|z_{n}\right|} \int_{|w|<\frac{1}{2}} \frac{1}{|w|}\left|\varphi_{z_{n}}^{\prime}(w)\right|^{2} d m(w) \\
& =2 \sum_{n=1}^{\infty} \frac{1}{1-\left|z_{n}\right|} \int_{|w|<\frac{1}{2}} \frac{1}{|w|} \frac{\left(1-\left|z_{n}\right|^{2}\right)^{2}}{\left|1-\bar{z}_{n} w\right|^{4}} d m(w) \\
& \leq 2^{7} \sum_{n=1}^{\infty}\left(1-\left|z_{n}\right|\right) \int_{|w|<\frac{1}{2}} \frac{d m(w)}{|w|}<\infty .
\end{aligned}
$$

Hence, the assertion for $k=1$ is proved.

If the condition (8) holds for some $\alpha=1 / k$, where $k \geq 2$, then also (24) holds. Hence, we make an induction assumption that (13) holds for some $k \geq 1$, and so we have to prove that (13) holds also for the index $k+1$.

By [15, p. 44], we have

$$
\frac{B^{(k+1)}(z)}{B(z)}=\sum_{n=2}^{k+1} \sum^{*} M \prod_{j=1}^{m} \frac{B^{\left(n_{j}\right)}(z)}{B(z)}-\sum_{n=1}^{\infty} \sum_{j=0}^{k} \frac{\left(1-\left|z_{n}\right|^{2}\right) \bar{z}_{n}^{j} k !}{\left(1-\bar{z}_{n} z\right)^{j+1}\left(z_{n}-z\right)^{k-j+1}},
$$

where $M$ is a constant and the sum $\sum^{*}$ is taken over all $m$-tuples $\left(n_{1}, n_{2}, \ldots, n_{m}\right)$ for which $n_{1}+n_{2}+\cdots+n_{m}=k+1$, and $n_{j} \geq 1$ for $1 \leq j \leq m$. Next, we use the general form of the Hölder inequality with conjugate indices $p_{j}=\frac{k+1}{n_{j}}$ for which

$$
\frac{1}{p_{1}}+\frac{1}{p_{2}}+\cdots+\frac{1}{p_{m}}=1
$$

to conclude that

$$
\int_{\mathbb{D}} \prod_{j=1}^{m}\left|\frac{B^{\left(n_{j}\right)}(z)}{B(z)}\right|^{\frac{1}{k+1}} d m(z) \leq \prod_{j=1}^{m}\left(\int_{\mathbb{D}}\left|\frac{B^{\left(n_{j}\right)}(z)}{B(z)}\right|^{\frac{1}{n_{j}}} d m(z)\right)^{\frac{n_{j}}{k+1}}<\infty .
$$

Hence,

$$
\begin{aligned}
& \int_{\mathbb{D}}\left|\frac{B^{(k+1)}(z)}{B(z)}\right|^{\frac{1}{k+1}} d m(z) \\
& \quad \leq C_{2}+C_{3} \sum_{n=1}^{\infty} \sum_{j=0}^{k} \int_{\mathbb{D}}\left(\frac{1-\left|z_{n}\right|^{2}}{\left|1-\bar{z}_{n} z\right|^{k+2}\left|\varphi_{z_{n}}(z)\right|^{k-j+1}}\right)^{\frac{1}{k+1}} d m(z)
\end{aligned}
$$

where $C_{2}, C_{3}>0$ are constants. It suffices to consider the term with index $j=0$ since the associated integrand takes the largest value for every $z$ with index $j=0$, because 
$\left|\varphi_{z_{n}}(z)\right|<1$. Now,

$$
\begin{aligned}
& \int_{\mathbb{D}}\left(\frac{1-\left|z_{n}\right|^{2}}{\left|1-\bar{z}_{n} z\right|^{k+2}\left|\varphi_{z_{n}}(z)\right|^{k+1}}\right)^{\frac{1}{k+1}} d m(z)=\int_{\mathbb{D} \backslash \Delta\left(z_{n}, \frac{1}{2}\right)}+\int_{\Delta\left(z_{n}, \frac{1}{2}\right)} \\
& \quad \leq 2 \int_{\mathbb{D}}\left(\frac{1-\left|z_{n}\right|^{2}}{\left|1-\bar{z}_{n} z\right|^{k+2}}\right)^{\frac{1}{k+1}} d m(z)+\frac{2^{\frac{1}{k+1}}}{1-\left|z_{n}\right|} \int_{\Delta\left(z_{n}, \frac{1}{2}\right)} \frac{d m(z)}{\left|\varphi_{z_{n}}(z)\right|} \\
& \quad \leq 2\left(1-\left|z_{n}\right|^{2}\right)^{\frac{1}{k+1}} \int_{\mathbb{D}} \frac{d m(z)}{\left|1-\bar{z}_{n} z\right|^{\frac{k+2}{k+1}}}+\frac{2^{\frac{1}{k+1}}}{1-\left|z_{n}\right|} \int_{|w|<\frac{1}{2}} \frac{1}{|w|}\left|\varphi_{z_{n}}^{\prime}(z)\right|^{2} d m(w) \\
& \quad \leq C_{4}\left(1-\left|z_{n}\right|\right)^{\frac{1}{k+1}},
\end{aligned}
$$

where $C_{4}>0$ is a constant. This proves (13) for the index $k+1$, and hence the assertion follows by the principle of mathematical induction.

REMARK. For $f \in H(\mathbb{D}), k \in \mathbb{N}$ and $p>0$, we recall from [14, pp. 179-180] the asymptotic comparability

$$
\int_{0}^{2 \pi}\left|D^{k} f\left(r e^{i \theta}\right)\right|^{p} d \theta \asymp \int_{0}^{2 \pi}\left|f^{(k)}\left(r e^{i \theta}\right)\right|^{p} d \theta, \quad r \rightarrow 1^{-},
$$

where $D^{k} f$ is the fractional derivative of $f$ of order $k$. Now Lemma 4 can be seen as an improvement of a special case of [14, Theorem 3.1. (2)(3)], where $\alpha=0, \beta=k$, $p=1 / k$, and the fractional derivative is replaced with the usual derivative of the same order $k$.

We proceed to prove Theorem 4 . Let $B$ be a Blaschke product having zeros at the points $z_{n}$ satisfying the given assumptions. Then, by [5, p. 192], the sequence $\left\{z_{n}\right\}_{n=1}^{\infty}$ is interpolating for some Bergman space $B^{p}$ with $p>1$. Hence we can find a function $g \in H(\mathbb{D})$ satisfying (9) such that $g^{\prime} \in B^{p}$ and that $f=B e^{g}$ solves (2), where $A(z)$ is given by $(10)$ and belongs to $H(\mathbb{D})$. On the other hand, by [5, p. 80], it is clear that $g^{\prime \prime} \in B^{\frac{1}{2}}$. Thus the assertion follows by (21), the Hölder inequality and Lemma 4 .

5. Proof of Theorem 5. Let $g(z)=\log (1-z)$ and $h(z)=z /(1-z)$. We see that the functions

$$
f_{j}(z)=\exp \left(g(z)+(-1)^{j} h(z)\right), \quad j=1,2,
$$

are linearly independent solutions of (2), where

$$
A(z)=-\left(g^{\prime \prime}(z)+g^{\prime}(z)^{2}+h^{\prime}(z)^{2}\right)=-(1-z)^{-4}
$$


belongs to $H(\mathbb{D})$ and satisfies

$$
\begin{aligned}
\int_{D(0, r)}|A(z)|^{\frac{1}{2}} d m(z) & =\int_{0}^{r} \int_{0}^{2 \pi} \frac{1}{\left|1-t e^{i \theta}\right|^{2}} d \theta t d t \\
& =\int_{0}^{r} \frac{1}{1-t^{2}} \int_{0}^{2 \pi} \frac{1-t^{2}}{\left|1-t e^{i \theta}\right|^{2}} d \theta t d t \\
& =\pi \int_{0}^{r} \frac{2 t}{1-t^{2}} d t=\pi \log \frac{1}{1-r^{2}}
\end{aligned}
$$

Thus $A \notin B^{\frac{1}{2}}$. Since the base functions $f_{1}$ and $f_{2}$ have no zeros, we turn our attention to their linear combinations. For simplicity, we consider the special case $f=f_{2}-C f_{1}$, $C>0$, leaving the general solution $c f_{1}+d f_{2}, c, d \in \mathbb{C}$, to the reader.

By the $2 \pi i$-periodicity of the exponential function, the solution $f$ has zeros exactly at points $z_{n}$ satisfying $h\left(z_{n}\right)=D+\pi n i$, where $D=(\log C) / 2$. Since $h$ maps $\mathbb{D}$ onto the half-plane $\Re z>-1 / 2$, we need to assume $C>1 / e$ in order for the solution $f$ to have zeros in the first place. Under the assumption $C>1 / e$ the zeros of $f$ are precisely at the points

$$
z_{n}=\frac{D+\pi n i}{1+D+\pi n i}=\frac{D(1+D)+\pi^{2} n^{2}+\pi n i}{(1+D)^{2}+\pi^{2} n^{2}}, \quad n \in \mathbb{Z} .
$$

We see that

$$
\sum_{n=-\infty}^{\infty}\left(1-\left|z_{n}\right|^{2}\right)^{\alpha}=\sum_{n=-\infty}^{\infty}\left(\frac{1+2 D}{(1+D)^{2}+\pi^{2} n^{2}}\right)^{\alpha}<\infty
$$

holds for every $\alpha>1 / 2$. In addition, (8) is not valid for $\alpha \in(0,1 / 2]$. On the other hand, by a direct calculation, we obtain

$$
1-\left|\frac{z_{n}-z_{k}}{1-\bar{z}_{n} z_{k}}\right|^{2}=\frac{(1+2 D)^{2}}{\pi^{2}(n-k)^{2}+(1+2 D)^{2}},
$$

so that

$$
\begin{aligned}
\sum_{n \neq k}\left(1-\left|\frac{z_{n}-z_{k}}{1-\bar{z}_{n} z_{k}}\right|\right) & \leq \frac{(1+2 D)^{2}}{\pi^{2}} \sum_{n \neq k} \frac{1}{(n-k)^{2}} \\
& =\frac{2(1+2 D)^{2}}{\pi^{2}} \sum_{m=1}^{\infty} \frac{1}{m^{2}}=\frac{(1+2 D)^{2}}{3}
\end{aligned}
$$

for every $k \in \mathbb{Z}$. In particular, the upper bound is independent on $k$. This implies (7), and so the sequence $\left\{z_{n}\right\}_{n \in \mathbb{Z}}$ is uniformly separated.

Since $\left\{z_{n}\right\}_{n \in \mathbb{Z}}$ is uniformly separated, it is also separated. From (26) we see that the separation constant $\delta$ defined in (6) is $\delta=\pi / \sqrt{\pi^{2}+(1+2 D)^{2}}$. If the constant $C>1 / e$ is chosen close enough to $1 / e$, then this $\delta$ clearly satisfies the condition (12).

Finally, since $e^{h} \in N$, it follows that every solution $f$ belongs to $N$. Moreover, the functions $g, h$ as well as their derivatives belong to the union of all Hardy spaces, and hence in $N$, so that $f^{(n)} \in N$ for all $n \in \mathbb{N}$. 
ACKNOWLEDGEMENT. The research reported in this paper was supported in part by the Academy of Finland, Project \#268009, the Faculty of Science and Forestry of the University of Eastern Finland, Project \#930349, and the Finnish Academy of Science and Letters.

\section{REFERENCES}

1. S. Buckley, P. Koskela and D. Vukotić, Fractional integration, differentiation, and weighted Bergman spaces, Math. Proc. Camb. Phil. Soc. 126(2) (1999), 369-385.

2. L. Carleson, An interpolation problem for bounded analytic functions, Am. J. Math. 80 (1958), 921-930.

3. M. Chuaqui, J. Gröhn, J. Heittokangas and J. Rättyä, Zero separation results for solutions of second order linear differential equations, Adv. Math. 245 (2013), 382-422.

4. P. Duren, Theory of $H^{p}$ spaces (Academic Press, New York, 1970).

5. P. Duren and A. Schuster, Bergman Spaces, Mathematical Surveys and Monographs, vol. 100 (American Mathematical Soc., Providence, 2004).

6. E. Fricain and J. Mashreghi, Integral means of the derivatives of Blaschke products, Glasg. Math. J. 50(2) (2008), 233-249.

7. Y. Gotoh, On integral means of the derivatives of Blaschke products, Kodai Math. J. 30(1) (2007), 147-155.

8. J. Gröhn and J. Heittokangas, New findings on Bank-Sauer approach in oscillation theory, Constr. Approx. 35 (2012), 345-361.

9. H. Hedenmalm, B. Korenblum and K. Zhu, Theory of Bergman spaces, Graduate Texts in Mathematics, vol. 199 (Springer, New York, 2000).

10. J. Heittokangas, A survey on Blaschke-oscillatory differential equations, with updates, in Blaschke products and their applications, Fields Inst. Commun. vol. 65 (Fricain E. and Mashreghi J., Editors) (Springer, New York, 2013), 43-98.

11. J. Heittokangas, Solutions of $f^{\prime \prime}+A(z) f=0$ in the unit disc having Blaschke sequences as the zeros, Comput. Methods Funct. Theory 5(1) (2005), 49-63.

12. J. Heittokangas and I. Laine, Solutions of $f^{\prime \prime}+A(z) f=0$ with prescribed sequences of zeros, Acta Math. Univ. Comen. 124(2) (2005), 287-307.

13. J. Heittokangas, R. Korhonen and J. Rättyä, Linear differential equations with coefficients in weighted Bergman and Hardy spaces, Trans. Am. Math. Soc. 360 (2008), 10351055.

14. H. Kim, Derivatives of Blaschke products, Pac. J. Math. 114(1) (1984), 175-190.

15. C. Linden, $H^{p}$-derivatives of Blaschke products, Mich. Math. J. 23 (1976), 43-51.

16. Z. Nehari, The Schwarzian derivative and Schlicht functions, Bull. Am. Math. Soc. 55 (1949), 545-551.

17. Ch. Pommerenke, On the mean growth of the solutions of complex linear differential equations in the disk, Complex Var. Theory Appl. 1(1) (1982), 23-38.

18. D. Protas, Blaschke products with derivative in $H^{p}$ and $B^{p}$, Mich. Math. J. 20 (1973), 393-396.

19. V. Šeda, On some properties of solutions of the differential equation $y^{\prime \prime}+A y=0$ with solutions having the prescribed zeros, Acta Fac. Nat. Univ. Comen. Math. 4 (1959), 223-253. (Slovak)

20. S. Yamashita, Gap series and $\alpha$-Bloch functions, Yokohama Math. J. 28 (1980), 31-36. 